innovare

Ciencia y Tecnología

\title{
HÁBITOS DE ESTUDIO Y RENDIMIENTO ACADÉMICO EN ESTUDIANTES UNIVERSITARIOS
}

\author{
Omar Bonerge Pineda Lezama, Nelly Jeannette Alcántara Galdámez ${ }^{1}$ \\ Facultad de Ingeniería y Arquitectura, Universidad Tecnológica Centroamericana (UNITEC), \\ Campus de San Pedro Sula
}

(Recibido: Enero, 2017/ Aceptado: Diciembre, 2017)

\section{Resumen}

Esta investigación tuvo como propósito determinar la relación existente entre los hábitos de estudio y el rendimiento académico de los estudiantes de álgebra de una institución universitaria privada de San Pedro Sula, durante el año académico 2016. El estudio fue desarrollado siguiendo un enfoque cuantitativo, de alcance descriptivo-correlacional y diseño transversal. La muestra fue probabilística no experimental constituida por 238 estudiantes. El instrumento de recogida de información empleado fue el de hábitos de estudio de Mena, Golbach y Veliz (2009), proporcionando una confiabilidad de 0.89 que evalúa cinco dimensiones: organización de horarios, metodología de estudio, comportamiento frente a un examen, factores internos y motivaciones para aprender; y disposición para enfrentarse a problemas algebraicos. Los resultados de la investigación demuestran la existencia de una relación estadísticamente significativa, entre los niveles de hábitos de estudio y los niveles del rendimiento académico de los estudiantes que cursan álgebra

Palabras Claves: Hábitos de estudio, rendimiento académico.

\begin{abstract}
The purpose of this research was to determine the relationship between study habits and academic performance of algebra students at a private university in San Pedro Sula, during the 2016 academic year. The study was developed following a quantitative, scope approach descriptive-correlational and transversal design. The sample was non-experimental probabilistic constituted by 238 students. The questionnaire used to collect the data was, the study habits of Mena, Golbach and Veliz (2009), providing a reliability of 0.89 that evaluates five dimensions: schedule organization, study methodology, behavior when taking an exam, internal factors and motivations to learn and willingness to face algebraic problems. The results of the research demonstrate the existence of a statistically significant relationship between the levels of study habits and the levels of academic performance of students studying algebra.
\end{abstract}

Keywords: Study habits, academic performance.

\footnotetext{
${ }^{1}$ Autor para correspondencia. Email: omarpineda@unitec.edu, n.alcantara@unitec.edu
} 


\section{Introducción}

El interés por los hábitos de estudio de los alumnos universitarios se ha convertido en un tema de actualidad tanto a nivel nacional como internacional debido al alto porcentaje de reprobación (Villegas, Muñoz, \& Villegas, 2016). Los autores; Lammers, Onwuegbuzie y Slate (2001), citado por Mena, Golbach y Veliz en 2009; establecen que "los hábitos, las técnicas y además las actitudes de estudio de los alumnos son factores que se relacionan significativamente en sus rendimientos académicos" (p.4). Otros autores, como Oñate y Gómez (1991) citado por Mena, Golbach y Veliz en 2009; consideran que: "los hábitos de estudio influyen en el rendimiento académico y además en el desempeño profesional" (p.5).

Uno de los mayores desafíos que enfrenta actualmente el sistema educativo nacional es el bajo rendimiento académico que presentan los estudiantes en áreas del conocimiento como español y matemáticas (Peña, 2014). El nivel universitario no es ajeno a esta problemática, en la que asignaturas específicas como álgebra tienen altos niveles de reprobación.

Para los docentes y para muchos estudiantes de primer ingreso la aprobación de la clase de álgebra representa un reto debido a la carencia de cimientos básicos en esta área del conocimiento, así como a la predisposición hacia la materia por considerarla difícil. Son varios los factores que pueden asociarse al nivel de rendimiento del estudiante, siendo uno de ellos los hábitos de estudio que posee el educando. Los autores Martínez y Torres (2005) citado por Villegas, Muñoz y Villegas (2009, p.34) indican que "no todos los estudiantes enfrentan con buen suceso los desafíos de la vida universitaria que implica una mayor dedicación al estudio y una mayor organización del trabajo académico", entre otros aspectos.

Jara et al. (2008, párr. 6) indican que "las causas del bajo rendimiento de los estudiantes están relacionadas con la dificultad para el auto-aprendizaje, escasos conocimientos básicos sobre las ciencias; así como la constante práctica de un aprendizaje memorístico". El bajo rendimiento académico del estudiante refleja una situación que afecta a toda la comunidad estudiantil: estudiantes, padres, maestros y en consecuencia a toda la sociedad.

Con esta investigación se pretende contestar la siguiente pregunta: ¿Cuál es el grado de asociación entre los hábitos de estudio y el rendimiento académico del estudiante universitario de San Pedro Sula?

\section{Objetivos}

\subsection{Objetivo General}

Establecer la relación entre los hábitos de estudio y el rendimiento académico en estudiantes universitarios matriculados en el curso de álgebra.

\subsection{Objetivos Específicos}


- Clasificar los hábitos de estudio de los estudiantes universitarios matriculados en el curso de álgebra.

- Categorizar el rendimiento académico de los estudiantes universitarios matriculados en el curso de álgebra.

- Medir el grado de asociación entre los hábitos de estudio y el rendimiento académico de los estudiantes universitarios matriculados en el curso de álgebra.

\section{Marco Teórico}

Este apartado señala la fundamentación teórica en la que se enmarca la presente investigación. El desarrollo del mismo se realiza de esta manera: hábitos de estudio, rendimiento académico y finalmente; relación entre hábitos de estudio y rendimiento académico.

De acuerdo a Cutz (2003) citado por Ríos y Ramos (2013, p.24), los hábitos de estudio son: "la repetición del acto de estudiar realizado bajo condiciones ambientales de espacio, tiempo y características iguales". Según Negrete (2009), citado por Gómez Argentina (2013) los hábitos de estudio son: "las actividades o experiencias que se efectúan continuamente con el fin de obtener un mayor beneficio en el proceso de aprendizaje" (p.22).

Dentro de los trabajos realizados para estudiar los hábitos de estudio figura el desarrollado por Acevedo, Torres y Tirado (2015), con estudiantes universitarios de Cartagena, Colombia donde se evaluaron entre otros aspectos: los factores ambientales encontrando que la mayoría de los estudiantes no tienen un lugar fijo para estudiar; otro factor evaluado corresponde a la organización del tiempo donde se encontró que la mayoría de los estudiantes no distribuyen su tiempo de estudio semanalmente. Sobre los métodos de estudio se concluyó que la gran mayoría de estudiantes no realizan una síntesis o resumen de lo estudiado. Acerca de la realización de exámenes se halló que la mayoría de los estudiantes no leen detenidamente las instrucciones de los mismos.

En la investigación sobre los hábitos de estudio de estudiantes, de una universidad peruana, determinaron que la mayoría de estudiantes dice leer en forma ocasional o semanalmente. Siendo el lugar más frecuente de lectura la casa, en contraste con un nivel relativamente bajo de alumnos que leen en la biblioteca. Asimismo, el resultado global de hábitos de estudio obtuvo un nivel bajo, hallándose puntajes negativos elevados, entre $54 \%$ y $42 \%$, en las cuatro dimensiones que evaluaron: hábitos de concentración, distribución del tiempo y relaciones sociales durante el estudio, técnicas para leer y tomar apuntes y; hábitos y actitudes generales de trabajo (Picasso-Pozo, Villanelo-Nimapaytan, \& Bedoya-Arboleda, 2015).

En el estudio llevado a cabo con estudiantes del curso de química en una universidad mexicana se investigaron los hábitos de estudio de los alumnos en dos momentos diferentes antes de recibir una capacitación sobre hábitos de estudio y posterior a la capacitación. El resultado indica que los estudiantes mejoraron en la organización de su tiempo de estudio, 
pero persistían como factores aún por erradicar la falta de concentración al estudiar, la escasa disciplina para el estudio y la dificultad para relacionar lo aprendido con otras áreas del conocimiento (Reyes \& Obaya, 2008).

Jara et al. (2008) indican que "el rendimiento académico es la capacidad de respuesta que tiene un individuo a estímulos, objetivos y propósitos educativos previamente establecidos" (p.2). Por otro lado, los autores Fernández y Rubal (2014) definen el rendimiento académico como "un indicador del nivel de aprendizaje alcanzado por el alumno" (p.2). Un rendimiento académico bajo indica que el estudiante no ha adquirido de forma adecuada y completa los conocimientos y que no posee las técnicas y habilidades necesarias para la solución de problemas relativos a la materia de estudio.

Según Garbanzo Vargas (2007), el rendimiento académico es una medida numérica del desempeño del estudiante de todas las actividades académicas desarrolladas durante un curso, sin embargo, hace énfasis acerca de la complejidad que conlleva el rendimiento académico y resalta tres perspectivas de diferente índole: personal, social e institucional. Destaca dentro de la perspectiva personal: la motivación del estudiante, la formación académica previa y la asistencia a clases; entre otros. En cuanto a la perspectiva social destaca el nivel educativo de la madre y el entorno de la familia. Y finalmente plantea dentro de la perspectiva institucional: el ambiente estudiantil y la relación estudianteprofesor, entro otros aspectos. El estudio concluye que el rendimiento académico refleja la calidad educativa de la institución o en última instancia del país (Garbanzo Vargas, 2007).

A continuación, se presentan los resultados de investigaciones llevadas a cabo para estudiar la relación entre los hábitos de estudio y el rendimiento académico. El trabajo desarrollado con 234 estudiantes universitarios de la ciudad Puerto Maldonado en Perú; determinó que existe un significativo grado de asociación entre los hábitos de estudio y el rendimiento académico (Ríos \& Ramos, 2013). En esta investigación, los autores encuentran que únicamente el 3.4\% de los estudiantes encuestados poseen un nivel bueno de hábitos de estudio, en contraste con un $96.6 \%$ que obtuvo un nivel regular y malo. Determinaron debilidades en la planificación, organización; y la comprensión y síntesis de la información.

Asimismo, en el estudio llevado a cabo en la Universidad de Tucumán, Argentina en el año 2009; donde se demostró de forma cuantitativa la relación existente los hábitos de estudio y el rendimiento académico. Para tal efecto clasificaron los resultados de las encuestas en dos grupos de estudiantes: los que poseen buenos hábitos de estudio y los que no poseen buenos hábitos de estudio; en ambos casos encontraron una correlación con el desempeño obtenido; es decir rendimiento académico alto relacionado a buenos hábitos de estudio y viceversa (Mena, Golbach, \& Véliz, 2009).

El estudio desarrollado en el año 2010, con casi 80 estudiantes de la carrera de enfermería en México, inicialmente determinó los hábitos de estudio por medio del "inventario de hábitos de estudio" creado en 2003 por Gilbert Wreenn. Este determina los hábitos de estudio agrupándolos en cuatro escalas: las técnicas de lectura y apuntes, las prácticas de concentración, la distribución del tiempo de estudio y las actitudes generales para estudiar. En este estudio se relacionó cada una de las escalas anteriores con el nivel de rendimiento encontrando que, a mejores hábitos de estudio, el rendimiento académico es mayor. La 
evaluación del grado de vinculación de la escala global de hábitos de estudio y el nivel de rendimiento obtuvo un nivel de relación significativo de forma estadística (Cruz Núñez \& Quiñones Urquijo, 2016).

Los factores que se asocian a un bajo rendimiento académico han sido objeto de estudio resultando uno de estos; los hábitos de estudio. Indican de forma concreta las siguientes características: el estudiante hace un mayor uso de estrategias de aprendizaje memorísticas, fallas en la capacidad de reflexión y de análisis crítico, escaso tiempo dedicado al estudio y la preparación se limita a un día antes del examen (Jara, y otros, 2008).

Asimismo, se han efectuado estudios para determinar los factores que se asocian con altos niveles de rendimiento en el área de Matemáticas, destacando los hábitos de estudio del estudiante. Específicamente resaltan en los resultados: la costumbre de practicar ejercicios de forma individual, la práctica de resolver problemas en pequeños grupos, la rutina de prestar atención a la explicación del profesor durante la clase, entre otros aspectos (Barbero, Holgado, Vila, \& Chacón, 2007).

Según Contreras, Caballero, Palacio y Pérez (2008) el bajo rendimiento académico se debe analizar desde una perspectiva multi-causal de tal forma que aborde de manera integral factores ligados al comportamiento del estudiante, a su personalidad, y a la naturaleza afectiva entre otros. Indican en su estudio que cada factor se debería abordar desde tres perspectivas: personal, familiar y social. Sin embargo, destacan en su estudio los factores relacionados con el comportamiento del estudiante, más concretamente, los hábitos de estudio del estudiante.

En la investigación llevada a cabo con estudiantes universitarios de primer ingreso se determinó que la mayoría de los estudiantes analizados no usan apropiadas técnicas de estudio y no distribuyen su tiempo de forma adecuada. Dentro de los aspectos cognitivos que se relacionan con el bajo rendimiento académico identifican que los estudiantes de primer ingreso en el ámbito universitario en su mayoría no entienden los contenidos de asignaturas que utilizan números y las que utilizan la lógica (Contreras, Caballero, Palacio, \& Pérez, 2008).

El trabajo desarrollado por Fernández y Rubal (2014), obtiene como resultado que los estudiantes poseen muy buenos hábitos de estudio, sin embargo, el resultado del rendimiento académico es regular, lo cual indica que además intervienen otros factores como el estado emocional, el estado físico, la edad cronológica del estudiante, nivel de inteligencia, entre otros. Además, señalan que este tipo de estudios puede verse afectado por la deseabilidad social por parte de los entrevistados en este caso los estudiantes.

\section{Metodología}

Se plantea la siguiente hipótesis de investigación: H1: Existe relación entre los hábitos de estudio y el nivel de rendimiento académico de los estudiantes universitarios. La investigación tiene un enfoque cuantitativo con un alcance correlacional y un diseño de tipo transversal. Hernández, Fernández y Baptista (2014) indican que "el diseño 
transversal se caracteriza porque los datos se recolectan en un solo momento y tiempo único" (p.154).

La recolección de los datos se realizó mediante la construcción de un cuestionario dividido en dos partes. La primera parte se centró en las variables que recolectan información de tipo general de los alumnos, tales como sexo, edad, número de cuenta, carrera en la que se encuentra matriculado y rendimiento académico en el primer parcial de la asignatura de álgebra. La segunda parte ayudó a reunir datos sobre la percepción que tienen los estudiantes de los hábitos de estudio. Se trabajó con la escala Likert, adjudicándose a cada respuesta desde 5 puntos a las totalmente favorables, hasta 1 punto a las totalmente desfavorables, ya que los alumnos contaron con 5 opciones para responder cada pregunta (Mc Millan \& Schumacher, 2005).

El instrumento contó con 24 ítems, de modo que el mínimo puntaje que podían obtener era de 24 puntos y el máximo de 120 puntos. El levantamiento de datos se efectúo en el mes de agosto del año 2016, la información recabada fue analizada haciendo uso de software Statistical Package for the Social Sciences (SPSS), versión 19 para Windows y el software PHStat versión 4.

El tipo de muestreo es probabilístico, se trabajó usando los pasos que proponen Fox y López (1981, pp.60-65). El primero, consistió en obtener el universo de los alumnos de álgebra, teniéndose un total de 325. En el segundo se fijó el criterio para seleccionar a los alumnos que pudieran responder el cuestionario, el cual consistió en que fueran estudiantes que se encontraran inscritos en el periodo julio-septiembre del 2016. Se establece un muestreo fijo, tal como lo define Namakforoosh (2000): "el muestreo fijo implica un muestreo de tamaño constante que se determina antes de recopilar los datos y no se modifica" (p.187). Se repartieron 300 cuestionarios y se hizo la invitación a los jóvenes a participar en la investigación. La muestra productora de datos fue de 238 alumnos que accedieron a responder el cuestionario. Aquí es necesario hacer énfasis en que un poco más de la tercera parte de los alumnos están cursando sus estudios en el turno matutino y el resto en el vespertino y nocturno. Se obtuvieron 200 cuestionarios en la mañana y tarde, y 38 en la noche.

Para obtener datos sobre la percepción de los estudiantes frente a los hábitos de estudio, que permitan identificar con claridad, la estructura de las dimensiones de los hábitos, y al mismo tiempo obtener el rendimiento académico del primer parcial de álgebra, el cuestionario elaborado se aplicó a un grupo de estudiantes que, durante el mes de agosto de 2016, recibieron el curso de álgebra en la Universidad Tecnológica Centroamericana de San Pedro Sula. Se realizó una sola encuesta por estudiante, a fin de garantizar que las percepciones capturadas sobre los hábitos de estudio fuesen independientes entre sí. Otra razón fue la de evitar generar malestar al estudiante realizándole más de una encuesta, lo que, de hacerse, aumentaría la posibilidad de que este responda en las siguientes encuestas de una manera no razonada. 


\section{Resultados y Hallazgos}

\subsection{Determinación del procedimiento del análisis factorial}

Antes de realizar la extracción de los factores se aplicó el contraste de esfericidad de Bartlett y la medida de adecuación muestral de Kaiser, Meyer y Olkin (KMO), con el propósito de verificar si los datos recolectados cumplían con las condiciones para la aplicación de un análisis factorial. Los resultados fueron los siguientes:

Tabla 1: Resultados del indicador KMO y prueba de Bartlett.

\begin{tabular}{|l|l|c|}
\hline \multicolumn{2}{|l|}{ Medida de adecuación muestral de Kaiser-Meyer-Olkin. } & 0.770 \\
\hline $\begin{array}{l}\text { Prueba de esfericidad de } \\
\text { Bartlett }\end{array}$ & Chi-cuadrado aproximado & 870.821 \\
\cline { 2 - 3 } & Grados de Libertad & 276 \\
\cline { 2 - 3 } & Nivel de Significancia & 0.000 \\
\hline
\end{tabular}

Fuente: Elaboración propia

La medida de adecuación muestral KMO es satisfactoria, se encuentra por encima del valor mínimo aceptable (0.5), al igual que la prueba de esfericidad de Bartlett, ya que el nivel de significación crítico que se obtuvo fue de 0.000 , tal como lo manifiestan Uriel y Aldas (2005, p.245). Por lo tanto, los resultados anteriores nos indican que tiene sentido aplicar el análisis factorial.

\subsection{Determinación del número de factores}

Para determinar la cantidad de dimensiones se debe emplear inicialmente la técnica estadística de Análisis de Componentes Principales (Johnson, 1998). Para seleccionar el método de extracción de factores se tendrán en cuenta los criterios establecidos por Malhotra (2004, p.245). En este método, el número de factores extraído se determinan de modo tal que el porcentaje acumulado de varianza extraída por los factores explique por lo menos el 60 por ciento de la varianza.

En la Tabla 2 se muestran todos los factores que se obtienen con el método de componentes principales. En esta tabla existen al menos cinco factores con valores propios iniciales superiores a uno, los cuales explican el $60.39 \%$ de la varianza, lo que puede interpretarse como un porcentaje aceptable según Malhotra (2004). Entonces esto permite expresar cada una de nuestras variables como una combinación lineal de estos cinco factores o dimensiones. 
Pineda Lezama \& Alcántara Galdámez / Innovare 6 (2) 19 - 34

Tabla 2: Variación total explicada

\begin{tabular}{|c|c|c|c|c|c|c|c|c|c|}
\hline \multirow{2}{*}{ 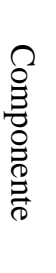 } & \multicolumn{3}{|c|}{ Auto-valores iniciales } & \multicolumn{3}{|c|}{$\begin{array}{l}\text { Sumas de las saturaciones } \\
\text { al cuadrado de la extracción }\end{array}$} & \multicolumn{3}{|c|}{$\begin{array}{c}\text { Suma de las saturaciones al } \\
\text { cuadrado de la rotación }\end{array}$} \\
\hline & $\stackrel{\overrightarrow{0}}{\stackrel{D}{\Xi}}$ & 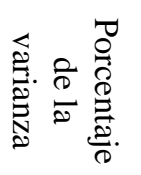 & 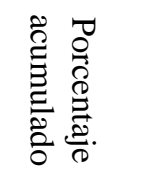 & $\begin{array}{l}\overrightarrow{0} \\
\stackrel{0}{\hat{\Xi}}\end{array}$ & 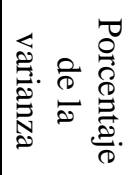 & 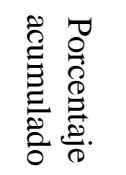 & $\stackrel{\overrightarrow{0}}{\stackrel{0}{D}}$ & 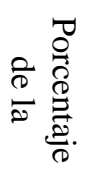 & 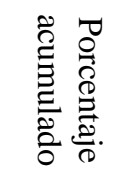 \\
\hline 1 & 7.365 & 30.689 & 30.689 & $\begin{array}{c}7.36 \\
5\end{array}$ & 30.689 & 30.689 & 4.518 & $\begin{array}{c}18.82 \\
6\end{array}$ & 18.826 \\
\hline 2 & 2.373 & 9.888 & 40.577 & $\begin{array}{c}2.37 \\
3\end{array}$ & 9.888 & 40.577 & 2.801 & $\begin{array}{c}11.66 \\
9\end{array}$ & 30.495 \\
\hline 3 & 2.116 & 8.816 & 49.394 & $\begin{array}{c}2.11 \\
6\end{array}$ & 8.816 & 49.394 & 2.661 & $\begin{array}{c}11.08 \\
9\end{array}$ & 41.584 \\
\hline 4 & 1.376 & 5.735 & 55.128 & $\begin{array}{c}1.37 \\
6\end{array}$ & 5.735 & 55.128 & 2.594 & $\begin{array}{c}10.81 \\
0\end{array}$ & 52.394 \\
\hline 5 & 1.264 & 5.265 & 60.393 & $\begin{array}{c}1.26 \\
4\end{array}$ & 5.265 & 60.393 & 1.920 & 7.999 & 60.393 \\
\hline 6 & 1.159 & 4.831 & 65.225 & & & & & & \\
\hline 7 & 1.049 & 4.369 & 69.593 & & & & & & \\
\hline 8 & .945 & 3.936 & 73.530 & & & & & & \\
\hline 9 & .832 & 3.465 & 76.995 & & & & & & \\
\hline 10 & .738 & 3.074 & 80.069 & & & & & & \\
\hline 11 & .664 & 2.765 & 82.834 & & & & & & \\
\hline 12 & .630 & 2.624 & 85.458 & & & & & & \\
\hline 13 & .494 & 2.058 & 87.516 & & & & & & \\
\hline 14 & .444 & 1.852 & 89.367 & & & & & & \\
\hline 15 & .434 & 1.809 & 91.176 & & & & & & \\
\hline 16 & .364 & 1.517 & 92.693 & & & & & & \\
\hline 17 & .341 & 1.420 & 94.113 & & & & & & \\
\hline 18 & .287 & 1.194 & 95.307 & & & & & & \\
\hline 19 & .257 & 1.070 & 96.377 & & & & & & \\
\hline 20 & .235 & .979 & 97.356 & & & & & & \\
\hline 21 & .194 & .808 & 98.164 & & & & & & \\
\hline 22 & .162 & .673 & 98.838 & & & & & & \\
\hline 23 & .153 & .635 & 99.473 & & & & & & \\
\hline 24 & .126 & .527 & 100.000 & & & & & & \\
\hline
\end{tabular}

Fuente: Elaboración propia 


\subsection{Rotación de factores}

La finalidad de la rotación es ayudar a interpretar el sentido y significado de los factores extraídos. En la Tabla 3 se presentan las dimensiones de los hábitos de estudio, la carga de los elementos luego de efectuar la rotación Varimax. Se consideraron como cargas significativas, aquellas con valor absoluto superior a 0.4 (Johnson, 1998). La cantidad de dimensiones es coherente con lo planteado por la literatura para los hábitos de estudio.

Tabla 3: Estructura de las dimensiones de los hábitos de estudio

\begin{tabular}{|c|c|c|c|c|c|}
\hline \multirow[t]{2}{*}{ Preguntas } & \multicolumn{5}{|c|}{ Componente } \\
\hline & 1 & 2 & 3 & 4 & 5 \\
\hline p17 & .142 & .057 & .159 & .768 & .308 \\
\hline $\mathrm{p} 21$ & .077 & .309 & .218 & .309 & .523 \\
\hline p18 & .142 & .142 & .020 & .563 & .238 \\
\hline p19 & .029 & .132 & -.093 & .729 & -.224 \\
\hline p6 & .293 & .463 & .236 & -.117 & -.432 \\
\hline p7 & -.039 & .695 & .394 & .109 & .104 \\
\hline $\mathrm{p} 1$ & .469 & .295 & .250 & .369 & .176 \\
\hline $\mathrm{p} 22$ & .315 & .339 & .393 & .211 & .559 \\
\hline $\mathrm{p} 8$ & .064 & .730 & .127 & .007 & .226 \\
\hline p13 & .062 & .086 & .913 & -.012 & .031 \\
\hline p9 & .359 & .727 & -.031 & .244 & -.044 \\
\hline p14 & .317 & .140 & .675 & -.070 & .138 \\
\hline p15 & .254 & .230 & .732 & .159 & -.069 \\
\hline $\mathrm{p} 2$ & .730 & .260 & -.004 & -.017 & .050 \\
\hline p3 & 697 & .313 & .155 & -.109 & -.087 \\
\hline $\mathrm{p} 4$ & .706 & .170 & .224 & -.282 & .031 \\
\hline $\mathrm{p} 5$ & 699 & .052 & .215 & .241 & -.044 \\
\hline $\mathrm{p} 16$ & .255 & -.012 & .666 & .177 & .228 \\
\hline $\mathrm{p} 23$ & .357 & .074 & .135 & -.076 & .702 \\
\hline $\mathrm{p} 24$ & .092 & .501 & .226 & .352 & .595 \\
\hline $\mathrm{p} 10$ & .078 & .602 & .151 & .353 & .175 \\
\hline p11 & -.036 & .623 & .116 & .415 & .122 \\
\hline p20 & .092 & .387 & -.138 & .292 & .262 \\
\hline $\mathrm{p} 12$ & -.108 & .647 & -.029 & .269 & .329 \\
\hline
\end{tabular}

Fuente: Elaboración propia

\subsection{Consistencia interna}

Con el propósito de analizar el carácter confiable de la estructura de las dimensiones de los hábitos de estudio y del cuestionario completo, se recurrió al coeficiente de consistencia 
interna Alpha de Cronbach (Landero \& González, 2007). Obteniéndose un valor general de 0.895 , ver tabla 4. Este resultado es satisfactorio de acuerdo a lo que plantea la literatura antes citada. El valor del coeficiente Alpha si el ítem es eliminado se mantiene en valores alrededor de 0.88 .

Tabla 4: Determinación del coeficiente Alpha de Cronbach

\begin{tabular}{|c|c|c|c|c|}
\hline Preguntas & $\begin{array}{c}\text { Media de la escala si } \\
\text { se elimina el } \\
\text { elemento }\end{array}$ & $\begin{array}{c}\text { Varianza de la escala } \\
\text { si se elimina el } \\
\text { elemento }\end{array}$ & $\begin{array}{l}\text { Correlación } \\
\text { elemento-total } \\
\text { corregida }\end{array}$ & $\begin{array}{c}\text { Alfa de Cronbach si } \\
\text { se elimina el } \\
\text { elemento }\end{array}$ \\
\hline $\mathrm{p} 1$ & 91.36 & 132.550 & .494 & .891 \\
\hline $\mathrm{p} 2$ & 91.13 & 133.588 & .481 & .892 \\
\hline p3 & 91.00 & 134.553 & .375 & .894 \\
\hline $\mathrm{p} 4$ & 90.84 & 138.212 & .206 & .897 \\
\hline p5 & 90.83 & 136.721 & .291 & .895 \\
\hline p6 & 91.86 & 128.045 & .475 & .892 \\
\hline p7 & 91.04 & 130.775 & .664 & .888 \\
\hline p8 & 90.90 & 130.805 & .556 & .890 \\
\hline p9 & 91.25 & 131.951 & .444 & .892 \\
\hline p10 & 90.69 & 133.559 & .397 & .893 \\
\hline $\mathrm{p} 11$ & 91.21 & 127.351 & .581 & .889 \\
\hline $\mathrm{p} 12$ & 91.05 & 131.760 & .491 & .891 \\
\hline $\mathrm{p} 13$ & 91.00 & 129.368 & .562 & .889 \\
\hline p14 & 90.81 & 132.396 & .525 & .891 \\
\hline p15 & 90.83 & 131.747 & .532 & .890 \\
\hline $\mathrm{p} 16$ & 90.81 & 133.764 & .471 & .892 \\
\hline p17 & 90.87 & 130.983 & .573 & .889 \\
\hline $\mathrm{p} 18$ & 91.00 & 129.079 & .585 & .889 \\
\hline p19 & 90.70 & 133.633 & .419 & .893 \\
\hline p20 & 91.77 & 127.260 & .533 & .890 \\
\hline $\mathrm{p} 21$ & 91.00 & 128.658 & .606 & .888 \\
\hline p22 & 91.13 & 129.957 & .556 & .890 \\
\hline p23 & 91.52 & 132.253 & .397 & .894 \\
\hline p24 & 91.01 & 132.066 & .481 & .891 \\
\hline
\end{tabular}

Fuente: Elaboración propia

\subsection{Rendimiento académico de los estudiantes}

El $61 \%$ de los estudiantes que participaron en el estudio son varones y el $39 \%$ mujeres; la edad promedio es de 18 años, con una desviación estándar de 2.05 años. Las edades de los alumnos varían entre 18 y 33 años. Como medida del rendimiento académico, se tomaron 
las calificaciones obtenidas en el primer parcial de álgebra, considerándose aprobado cuando esa calificación fue mayor o igual a $60 \%$.

En la Figura 1, se muestra la distribución porcentual de los alumnos respecto del rendimiento académico en el primer parcial de álgebra. Se observa que alrededor del $56 \%$ de los alumnos reprobaron el examen, y sólo un $43.8 \%$ de ellos aprobó dicho examen. Esto indica un alto grado de reprobados que lleva a plantearse la necesidad de analizar algunos de los factores que pueden incidir en esta problemática.

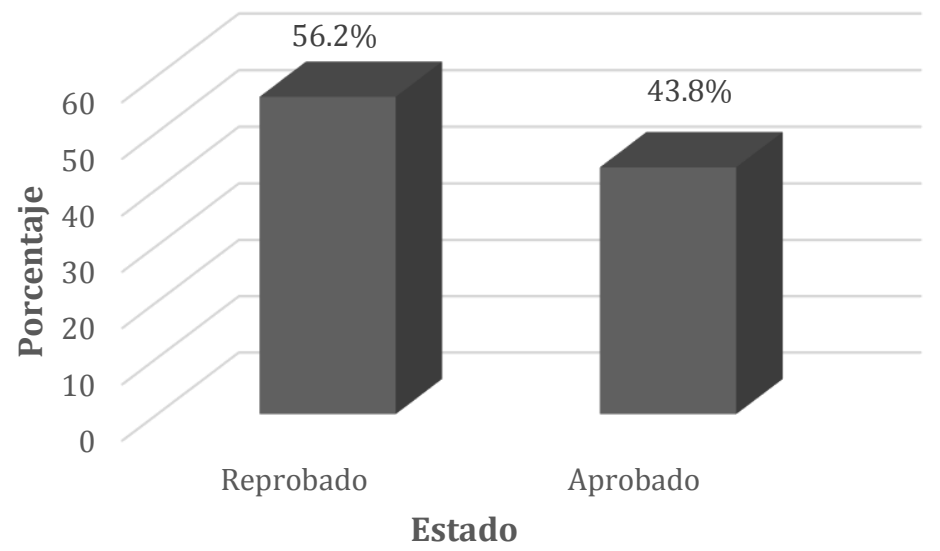

Figura 1: Distribución porcentual de 238 alumnos según el rendimiento académico en el primer parcial de álgebra.

Fuente: Elaboración propia.

En la Figura 2, se observa que del total de alumnos que aprobaron el parcial, más de la mitad de ellos obtuvo una calificación inferior a $60 \%$, además de ser muy bajo (15.97\%) el porcentaje de alumnos que aprobaron con puntaje óptimo. Sin embargo, se puede observar que existe un porcentaje considerable de alumnos con calificaciones no satisfactorias de 0 a 39 que representan el $28.57 \%$.

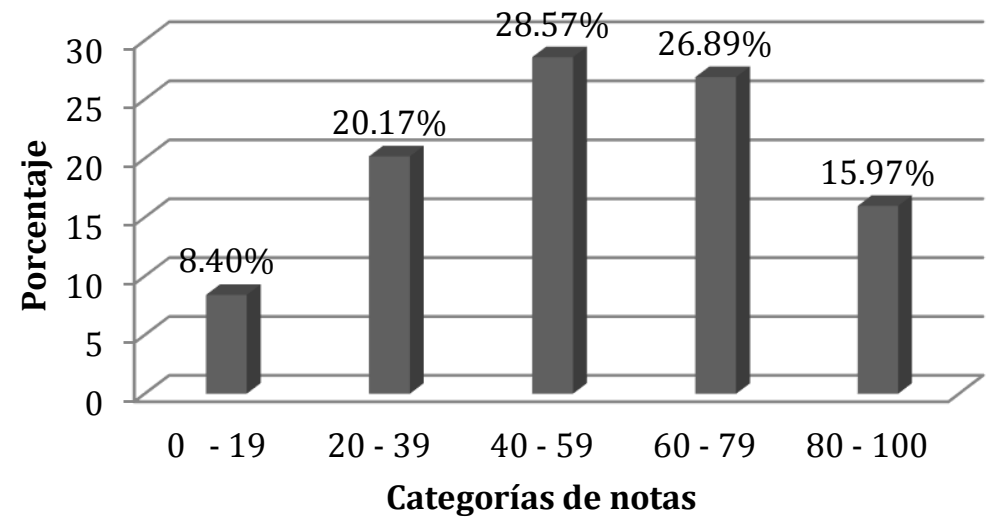

Figura 2: Distribución porcentual de 238 alumnos aprobados y reprobados en el primer parcial de álgebra según los puntajes distribuidos por categorías de notas.

Fuente: Elaboración propia. 


\subsection{Hábitos de estudio de los estudiantes}

Los indicadores que se tuvieron en cuenta en cada uno de los aspectos considerados para medir la variable hábitos de estudio se detallan en la Tabla 5 con sus resultados correspondientes. De acuerdo a la distribución de frecuencias de las puntuaciones totales obtenidas en la escala, se calcularon las medidas de tendencia central para la variable aditiva hábitos de estudio.

Tabla 5: Estadísticos descriptivos de la variable hábitos de estudio

\begin{tabular}{|l|l|}
\hline Media & 64.49 \\
\hline Rango resultante & 34 \\
\hline Puntuación más baja observada & 49 \\
\hline Puntuación más alta observada & 83 \\
\hline
\end{tabular}

Fuente: Elaboración propia.

Del procesamiento de la información presentada anteriormente, se identificó a los alumnos que poseían hábitos de estudios suficientes o favorables (GF) y a los que poseían pocos hábitos de estudio (GP). Y se construyeron dos clases de intervalos según los puntajes obtenidos en la escala. Como un criterio para separar a los alumnos en los dos grupos mencionados, se consideró la media (64.49), determinándose que un $39.92 \%$ de los alumnos poseían hábitos de estudios favorables.

En la Figura 3, se observa que del total de alumnos que cursan álgebra, más del $50 \%$ de ellos presentan pocos hábitos de estudio, además de ser muy bajo (39.92\%) el porcentaje de alumnos que tienen hábitos de estudio favorables, la cual representa una diferencia porcentual (20\%) muy considerable, por ende, se le debe de dar mucha importancia a estos alumnos que se encuentran clasificados en este grupo.

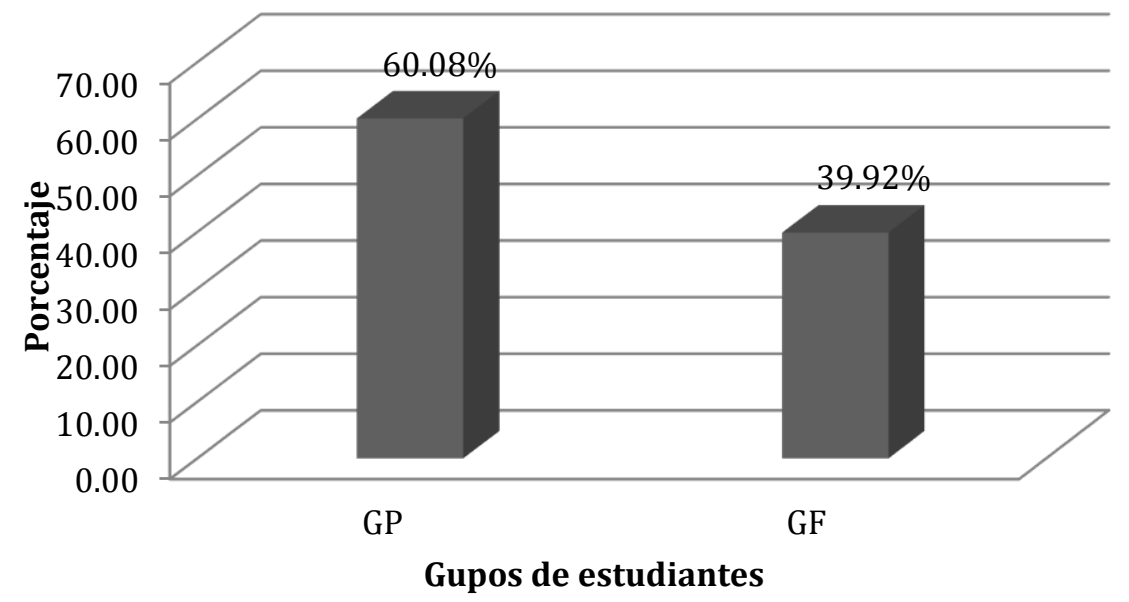

Figura 3: Distribución porcentual de frecuencias de la variable hábitos de estudio según los intervalos de puntajes obtenidos: GPH: [49 ; 64.49] y GHF: [64.49 ; 83].

Fuente: Elaboración propia. 


\subsection{El comparativo de los hábitos de estudio y rendimiento académico de los estudiantes}

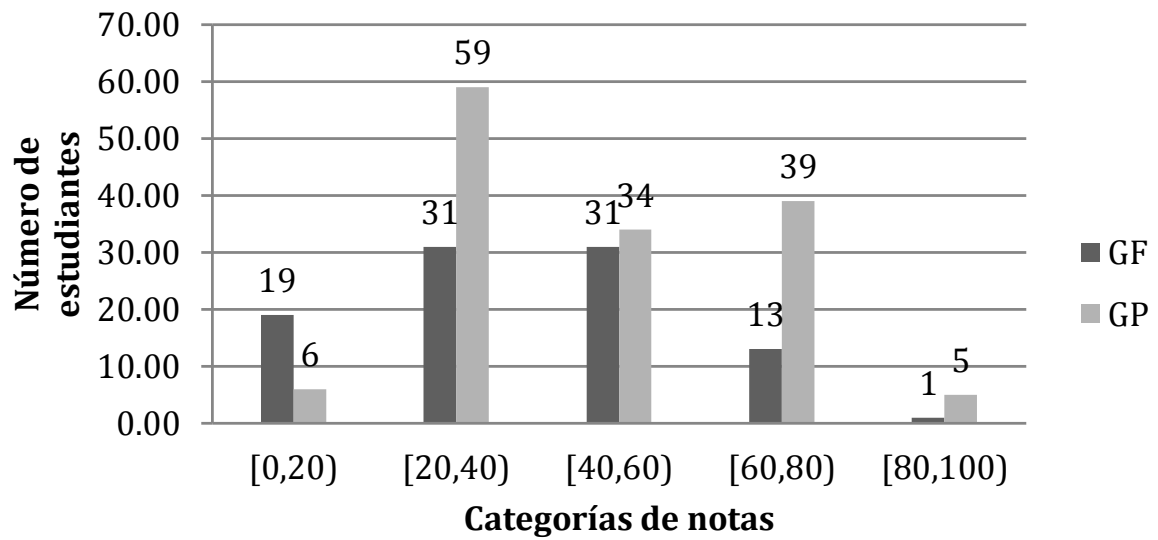

Figura 4: Distribución de frecuencias de la variable hábitos de estudio según el rendimiento académico en el primer parcial de álgebra.

Fuente: Elaboración propia.

La Figura 4, muestra el comportamiento de cada uno de los grupos (GF y GP) respecto del rendimiento académico. Es notable un bajo porcentaje de alumnos (5.8\%) que tienen hábitos de estudio favorables y aprobaron el parcial con una nota superior o igual a $60 \%$. Ese porcentaje va en disminución a medida que decrece el rendimiento académico, hasta llegar a un $34 \%$ de alumnos que con hábitos de estudio suficientes que desaprobaron el parcial.

El proceso a seguir es realizar una tabla de contingencia con las variables para posteriormente realizar la prueba de hipótesis. De acuerdo a la tabulación cruzada de las puntuaciones totales obtenidas en la escala de la variable hábitos de estudio y la variable rendimiento académico, se calcularon las observaciones al cruzar las categorías de los hábitos de estudios y las categorías de rendimiento académico que se pueden ver en la Tabla 6.

Tabla 6: Distribución de frecuencias de la variable hábito de estudio según el rendimiento académico en el primer parcial de álgebra.

\begin{tabular}{|c|c|c|c|}
\hline \multirow{2}{*}{ Rendimiento académico } & \multicolumn{2}{|c|}{ Hábitos de estudio } & \multirow{2}{*}{ Total } \\
\cline { 2 - 3 } & GF & GP & \\
\hline$[0,20)$ & 9.98 & 15.02 & 25 \\
\hline$[20,40)$ & 35.92 & 54.08 & 90 \\
\hline$[40,60)$ & 25.95 & 39.05 & 65 \\
\hline$[60,80)$ & 20.76 & 31.24 & 52 \\
\hline$[80,100)$ & 2.39 & 3.61 & 6 \\
\hline Total & 95 & 143 & 238 \\
\hline
\end{tabular}

Fuente: Elaboración propia.

Para comprobar el comportamiento en los dos grupos, se realizó una prueba de homogeneidad donde se obtuvo, $\chi 2=22.51121273$ y $\mathrm{p}=0.000158521$ para 4 grados de 
libertad, por lo que se concluyó a un nivel de significancia de 5\% que los hábitos de estudio de estos dos grupos afecta el rendimiento académico de los mismos, este resultado es congruente con los estudios realizados por Contreras, Caballero Palacio y Pérez (2008), de igual forma con Mena, Golbach y Veliz (2009), así como también con Ríos y Ramos (2013) y; Cruz, Núñez y Quiñones(2016).

\section{Conclusiones y Recomendaciones}

\subsection{Conclusiones}

Se clasificaron a los alumnos que poseían hábitos de estudios suficientes o favorables (GF) y a los que poseían pocos hábitos (GP) con un porcentaje aproximado de $40 \%$ y $60 \%$, respectivamente. El rendimiento académico de los estudiantes universitarios matriculados en el curso de álgebra, se categorizaron en cinco categorías de notas: [0,20), [20,40), $[40,60),[60,80)$ y $[80,100)$; con el fin de poder establecer una relación con los hábitos de estudio. Se comprobó una relación entre los hábitos de estudio y el rendimiento académico en estudiantes universitarios matriculados en el curso de álgebra, a un nivel de significancia de 5\%. Esto indica, que los hábitos de estudio de estos dos grupos están asociados con el rendimiento académico.

\subsection{Recomendaciones}

A través del análisis de este estudio se desprende que:

1. El instrumento de medición de hábitos de estudio y rendimiento académico referido en este trabajo, se muestra como un apoyo determinante útil para la práctica de la tutoría, el tutor debería de hacer uso del instrumento, y darle seguimiento al hacer conciencia en el estudiante los hábitos negativos y de esta manera tener un mejor rendimiento académico.

2. La detección oportuna de las clasificaciones de los hábitos de estudio permitirá a las autoridades académicas implementar medidas de apoyo que favorezcan el proceso de aprendizaje, disminuyan los niveles de reprobación; y con ello los riesgos de deserción, así como la desmotivación que causa la reprobación de un curso.

3. Es oportuno establecer mecanismos de apoyo y de orientación a los alumnos para su ingreso a la universidad, mostrándole las nuevas formas de estudio y estrategias de aprendizaje del nivel universitario.

\section{Bibliografía}

Acevedo, D., Torres, J., \& Tirado, D. (2015). Análisis de los hábitos de estudio y motivación para el aprendizaje a distancia en alumnos de ingeniería de sistemas de la universidad de Cartagena. Formación Universitaria, 60. 
Barbero, Holgado, Vila, \& Chacón. (2007). Actitudes, hábitos de estudio y rendimiento en Matemáticas: diferencias por género. Obtenido de Psicothema 2007. Vol. 19, no 3, pp. 413-421: http://www.unioviedo.es/reunido/index.php/PST/article/view/8548/8412

Contreras, Caballero, Palacio, \& Pérez. (2008). Factores asociados al fracaso académico en estudiantes universitarios de Barranquilla (Colombia). Obtenido de Psicología desde el Caribe. issn 0123-417X $\mathrm{N}^{\circ} \quad 22, \quad$ julio-diciembre 2008: http://www.scielo.org.co/pdf/psdc/n22/n22a08.pdf

Cruz Núñez, F., \& Quiñones Urquijo, A. (9 de Septiembre de 2016). Hábitos de estudio y rendimiento académico en enfermería, Poza Rica, Veracruz, México. Obtenido de Revista Electrónica Actualidades Investigativas en Educación, vol. 11, núm. 3,2011: https://www.researchgate.net/publication/237034876_HABITOS_DE_ESTUDIO_Y_RE NDIMIENTO_ACADEMICO_EN_ENFERMERIA_POZA_RICA_VERACRUZ_MEXI $\mathrm{CO}$

Fernández, M., \& Rubal, N. (2014). Los hábitos de estudio y el rendimiento académico en el primer año de la carrera de medicina. Obtenido de Congreso Virtual de Ciencias Morfológicas.: http://www.morfovirtual2014.sld.cu/index.php/Morfovirtual/2014/paper/download/198/2 83

Fox, \& López. (1981). Métodos y técnicas avanzadas de análisis de datos en ciencias del comportamiento. Barcelona.

Garbanzo Vargas, G. (2007). Factores asociados al rendimiento académico en estudiantes universitarios, una reflexión desde la calidad de la educación superior pública. Obtenido de Revista Educación 31(1), 43-63, ISSN: 0379-7082: http://dx.doi.org/10.15517/revedu.v31i1.1252

Gómez, A. (2013). Tesis: Relación entre hábitos de estudio y rendimiento académico en estudiantes. Quetzaltenango: Universidad Rafael Landívar.

Hernández, R., Fernández, C., \& Baptista, M. (2014). Metodología de la investigación . México: McGrawHill.

Jara, D., Velarde, H., Gordillo, G., Guerra, G., Arroyo, C., \& Figueroa, M. (2008). Factores influyentes en el rendimiento académico de estudiantes del primer año de medicina. Obtenido de An. Fac. Med. Lima.: http://www.scielo.org.pe/scielo.php?pid=S1025-55832008000300009\&script=sci_arttext

Johnson, D. E. (1998). Applied Multivariete Methods for Data Analysts. Duxbury Press.

Landero, R., \& González, M. (2007). Estadística con SPSS y Metodología de Investigación. México: Trillas.

Malhotra. (2004). Investigación de mercados: un enfoque aplicado. Educación. Pearson Educación.

Mc Millan, J., \& Schumacher, S. (2005). Investigación educativa: una introducción conceptual. Pearson Educación.

Mena, A., Golbach, M., \& Véliz, M. (2009). Influencia de los hábitos de estudio en el rendimiento de alumnos ingresantes. Obtenido de Facultad de Ciencias Económicas. Universidad Nacional de Tucumán: http://www.soarem.org.ar/Documentos/48\%20Mena.pdf 
Mena, A., Marta, G., \& Véliz, M. (s.f.). INFLUENCIA DE LOS HÁBITOS DE ESTUDIO EN ELRENDIMIENTO DE ALUMNOS INGRESANTES. Obtenido de http://www.soarem.org.ar/Documentos/48\%20Mena.pdf

Namakforoosh, M. (2000). Metodología de la investigación. México: Limusa Editores.

Peña, G. (7 de 04 de 2014). Honduras tiene el segundo peor rendimiento en educación de América Latina. El Heraldo, págs. http://www.elheraldo.hn/alfrente/564923-209/honduras-tiene-elsegundo-peor-rendimiento-en-educacion-de-america-latina.

Picasso-Pozo, M., Villanelo-Nimapaytan, M., \& Bedoya-Arboleda, L. (12 de Ene-Jun de 2015). Hábitos de lectura y estudio, y su relación con el rendimiento académico en estudiantes de Odontología de una universidad peruana. KIRU;12(1), 19-27. Obtenido de www.usmp.edu.pe/odonto/servicio/2015/Kiru_12-1_v_p19-27.pdf

Reyes, S., \& Obaya, V. (2008). Hábitos de estudio de alumnos de ingeniería agrícola y su impacto en el rendimiento obtenido en un curso de química básica. 2008;1(5):29-34. Formación Universitaria-Vol. $1 \quad N^{\circ} 5$, 29-34. Obtenido de Form Univ.: http://dx.doi.org/10.4067/S0718-50062008000500005

Ríos, V., \& Ramos, D. (p.23-32 de 2013). Hábitos de estudio y rendimiento académico de los estudiantes de ingeniería, ecoturismo y educación de la Universidad Nacional Amazónica de Madre de Dios, Ciudad Puerto Maldonado. Obtenido de El Ceprosimad, 2(1): http://ceprosimad.com/revista/habitos.pdf

Uriel, E., \& Aldas, J. (2005). Analisis multivariante aplicado. Thomson.

Villegas, C., Muñoz, F., \& Villegas, R. (11 de Septiembre de 2016). Hábitos de estudio de los alumnos en el área de Química Orgánica. Obtenido de Revista Biotecnia, Vol.XI,no3 Sept-Dic 2009: http://biotecnia.ojs.escire.net/index.php/biotecnia/article/viewFile/72/66 